\title{
Effects of tDCS on Bimanual Motor Skills: A Brief Review
}

\author{
Nils H. Pixa ${ }^{1 *}$ and Bettina Pollok ${ }^{2}$ \\ ${ }^{1}$ Department of Sport Psychology, Institute of Sports Science, Johannes Gutenberg-University Mainz, Mainz, Germany, \\ 2Institute of Clinical Neuroscience and Medical Psychology, Medical Faculty, Heinrich-Heine-University Düsseldorf, \\ Düsseldorf, Germany
}

\section{OPEN ACCESS}

Edited by:

Roberta Ferrucci, Fondazione IRCCS Ca' Granda Ospedale Maggiore Policlinico (IRCCS), Italy

Reviewed by: Wei Peng Teo, Deakin University, Australia Sean Kevin Meehan, University of Michigan, United States

*Correspondence:

Nils H. Pixa

pixa@uni-mainz.de

Received: 08 January 2018 Accepted: 16 March 2018 Published: 04 April 2018

Citation: Pixa NH and Pollok B (2018) Effects of tDCS on Bimanual Motor Skills: A Brief Review.

Front. Behav. Neurosci. 12:63. doi: 10.3389/fnbeh.2018.00063
Transcranial direct current stimulation (tDCS) is a non-invasive brain stimulation technique that allows the modulation of cortical excitability as well as neuroplastic reorganization using a weak constant current applied through the skull on the cerebral cortex. TDCS has been found to improve motor performance in general and motor learning in particular. However, these effects have been reported almost exclusively for unimanual motor tasks such as serial reaction time tasks, adaptation tasks, or visuomotor tracking. Despite the importance of bimanual actions in most activities of daily living, only few studies have investigated the effects of tDCS on bimanual motor skills. The objectives of this review article are: (i) to provide a concise overview of the few existing studies in this area; and (ii) to discuss the effects of tDCS on bimanual motor skills in healthy volunteers and patients suffering from neurological diseases. Despite considerable variations in stimulation protocols, the bimanual tasks employed, and study designs, the data suggest that tDCS has the potential to enhance bimanual motor skills. The findings imply that the effects of tDCS vary with task demands, such as complexity and the level of expertise of the participating volunteers. Nevertheless, optimized stimulation protocols tailored to bimanual tasks and individual performance considering the underlying neural substrates of task execution are required in order to probe the effectiveness of tDCS in greater detail, thus creating an opportunity to support motor recovery in neuro-rehabilitation.

Keywords: non-invasive brain stimulation, transcranial direct current stimulation, bimanual movements, bimanual coordination, motor learning and performance

\section{INTRODUCTION}

The non-invasive brain stimulation technique of transcranial direct current stimulation (tDCS) is a suitable method for modulating cortical excitability (Bindman et al., 1964; Nitsche and Paulus, 2001) as well as neuro-plastic reorganization (Fritsch et al., 2010; Hunter et al., 2013; Karabanov et al., 2015). Previous studies suggest that tDCS can be used to facilitate motor performance such as motor learning in healthy volunteers (Reis and Fritsch, 2011; Buch et al., 2017) and in patients suffering from neurological disorders (Bastani and Jaberzadeh, 2012; Flöel, 2014). Notably, the effects of tDCS on motor performance are almost exclusively evidenced by studies employing unimanual tasks. However, it is well known that in a large variety of daily activities both hands are required to accomplish required actions. Impaired bimanual skills due to neurological disease or age-related decline (for an overview see Maes et al., 2017) present a challenge to independent living. Despite the abundance of daily activities that require bimanual skills, only few studies have thus 
far investigated the effects of tDCS on bimanual performance. Considering the increasing number of tDCS studies and the high relevance of bimanual motor skills the purpose of this review article is to provide a preliminary systematic characterization of tDCS effects on bimanual actions.

\section{REVIEW CRITERIA}

The present review article focuses on studies addressing tDCS effects explicitly on bimanual motor performance, thus investigating bimanual motor outcome was the decisive criterion. To this end, a computer-based search of PubMed and Science Direct for articles from 2000 to 2017 was carried out in August 2017 using the following keywords: "bimanual" AND "transcranial direct current stimulation" OR "tDCS" OR "transcranial electrical stimulation" OR "non-invasive brain stimulation" OR "transcranial stimulation" and "coordination" AND "transcranial direct current stimulation" OR "tDCS" OR "transcranial electrical stimulation" OR "non-invasive brain stimulation" OR "transcranial stimulation". A total of 18 articles matched the criteria. Five articles did not directly investigate bimanual motor skills and were therefore excluded.

\section{TRANSCRANIAL DIRECT CURRENT STIMULATION}

TDCS allows the modulation of neural excitability (Bindman et al., 1962, 1964; Nitsche and Paulus, 2001). Using intensities of 0.5-2 milliampere (mA), a constant current is applied to the cerebral cortex via saline soaked sponges or gel-electrodes through the skull. Two electrodes with a surface area of $15-35 \mathrm{~cm}^{2}$ are used mostly, resulting in a current density of 0.014-0.133 mA/cm² (Ho et al., 2016; Woods et al., 2016). In high-definition tDCS (HD-tDCS), smaller (1-5 $\mathrm{cm}^{2}$ ) and often more than two electrodes (e.g., $4 \times 1$ configuration) are used to increase the current density and focality of the stimulated area (Villamar et al., 2013; Alam et al., 2016). Although its exact physiological mechanisms are still under debate, evidence exists that anodal tDCS increases the neural excitability of the stimulated area, while cathodal tDCS decreases it. Anodal stimulation shifts the resting membrane potential closer to the critical depolarization threshold, resulting in a higher excitability and spiking rate, while the opposite effect of tonic hyperpolarization is associated with cathodal stimulation (Bindman et al., 1962; Nitsche and Paulus, 2000; Romero Lauro et al., 2014). These effects have mainly been derived from the stimulation of the primary motor cortex (M1), although comparable effects have also been demonstrated in visual (Antal et al., 2004; Accornero et al., 2007), somatosensory (Dieckhöfer et al., 2006), and auditory cortices (Zaehle et al., 2011). Nevertheless, anatomical differences_in particular, the spatial orientation of neurons-need to be considered in order to understand the effects of tDCS on other brain areas (Accornero et al., 2007). Moreover, the effects of tDCS depend on several factors like current intensity, duration and timing of tDCS relative to the specific task (prior to vs. during; Stagg et al.,
2011; Batsikadze et al., 2013; Monte-Silva et al., 2013). The shape, size, number, and positions of the electrodes also determine the characteristics of tDCS such as distribution of the induced electrical field (Ho et al., 2016; Naros et al., 2016; Woods et al., 2016). Individual attributes of the participants (e.g., head anatomy, age) but also the excitability of the stimulated area are thought to influence the effects of tDCS yielding intraand inter-individual variability on physiological and behavioral measures (Ridding and Ziemann, 2010; Li et al., 2015; Opitz et al., 2015). Besides local effects on the stimulated brain area, tDCS is presumed to affect the excitability of functionally connected areas as well. Such remote effects can be in the same (Antal et al., 2011) or opposite (Stagg et al., 2009) direction as the effects in the stimulated area.

Changes of M1 excitability associated with tDCS have been found to persist after cessation of stimulation (Nitsche and Paulus, 2000, 2001). Such after-effects are associated with alterations in $\mathrm{N}$-methyl-d-aspartate (NMDA) receptors (Liebetanz et al., 2002) — at least within M1-yielding long-term potentiation (LTP)-like mechanisms. In motor learning, these effects seem to be strongest when tDCS is co-applied with motor training (Reis and Fritsch, 2011; Stagg et al., 2011) and applied over multiple days (Reis et al., 2009; Alonzo et al., 2012; Saucedo Marquez et al., 2013).

\section{tDCS AND BIMANUAL MOTOR SKILLS IN HEALTHY VOLUNTEERS}

Bimanual motor skills require a well-coordinated interplay between the upper limbs. Multiple brain areas are involved in orchestrating bimanual movements in space and time. Because the supplementary motor area (SMA) plays a pivotal role in such tasks (Swinnen, 2002; Debaere et al., 2004; Swinnen and Wenderoth, 2004; Swinnen and Gooijers, 2015), Carter et al. (2015) examined the effects of tDCS applied over the SMA on the stability, consistency, and transition of metronome-paced bimanual forearm movements from in- to anti-phase movements and vice versa. They reported improved stability and consistency as well as a delayed spontaneous transition from the anti- to the more stable in-phase pattern following anodal tDCS, while cathodal tDCS did not affect task performance (Carter et al., 2015). In line with this observation, anodal tDCS of the SMA was found to be associated with faster intentional switches from anti- to in-phase movements (Carter et al., 2017). These findings underline a central role of the SMA in the control of bimanual movements and suggest that tDCS represents a suitable method for the transient modulation of this process.

Typing a text on a keyboard requires bilateral wellcoordinated, skilled finger movements, and particularly practice to achieve successful task performance. Gomes-Osman and Field-Fote (2013) investigated the effects of anodal tDCS over bilateral M1 on a bimanual typing task. Task performance was assessed immediately before and after stimulation. After five consecutive training days, participants showed a larger improvement in the number of correctly-typed bimanual sequences following anodal tDCS as compared to sham 
stimulation. However, this effect vanished after a 1-week retention interval. Ciechanski and Kirton (2017) examined the effects of tDCS on dexterity using the Purdue Pegboard Test (PPT; Tiffin and Asher, 1948). The PPT measures uni- and bimanual hand functions such as placing the maximum number of pegs in the pegboard within $30 \mathrm{~s}$. While the bimanual task requires symmetrical movements of both hands, a second bimanual task (assembly) requires the asymmetrical (alternate) use of both hands to build a maximum number of small assemblies consisting of pins, collars and washers within $60 \mathrm{~s}$ (Desrosiers et al., 1995). Ciechanski and Kirton (2017) reported improved bimanual motor performance in healthy school children after training of the left hand with concurrent anodal tDCS applied to the contralateral M1 corresponding to C4. Interestingly, this effect was also present when cathodal tDCS was applied to the ipsilateral M1 (C3), but not after sham tDCS. While the symmetrical bimanual performance was facilitated, the more complex (asymmetrical) "assembly test" was not affected by either stimulation. After a retention interval of 6 weeks, improved task performance remained stable, suggesting enhanced motor consolidation associated with tDCS. Pixa et al. (2017b) also used the PPT to investigate the effect of multichannel HD-tDCS targeting bilateral M1 (see Table 1 for electrode positions). After a training period of 3 days with concurrent stimulation, larger cumulative performance gains in participants receiving anodal HD-tDCS compared to the sham-stimulated control group were found. Improved performance was indicated for the unimanual task of the dominant right hand and the bimanual PPT. Performance gains remained stable over a 1-week retention interval and further improvement was found for right-hand performance. As compared to sham tDCS, no significant differences were found for the unimanual left-hand task and-again-the more complex bimanual "assembly" task was not affected by tDCS (Pixa et al., 2017b). The same stimulation protocol was used to investigate effects of bilateral anodal HD-tDCS on the performance of a complex sequential bimanual stacking task (Pixa et al., 2017a). After 3 days of training in sport stacking (cup stacking) with concurrent anodal HD-tDCS, faster stacking performance compared to the sham group was found. This effect occurred for only one of the two required stacking formations (3-6-3), while only a statistical trend emerged for the more complex task-version (1-10-1). Re-testing after 1 week revealed sustained superior performance in the 3-6-3 stack for the anodal HD-tDCS group.

In two studies, Furuya et al. $(2013,2014 a)$ demonstrated that the effects of tDCS on bimanual motor performance depend on the subjects' level of expertise. While highly-trained expert pianists did not benefit from bilateral tDCS over M1 (anode right $\mathrm{M} 1$ and cathode left $\mathrm{M} 1$ and vice versa) in a bimanual finger typing task, the analysis revealed that only pianists who commenced piano training at an advanced age showed selectively improved performance following tDCS, irrespective of the stimulation protocol (Furuya et al., 2013). A second study using the same bimanual task, suggests that musically untrained volunteers (novices) significantly improved bimanual performance following tDCS, whereas expert pianists did not. Rather, the skilled finger movements of the pianists were found to be slightly deteriorated after verum stimulation compared to sham stimulation (Furuya et al., 2014a).

It should also be stressed that other studies have failed to provide evidence of significant stimulation effects on bimanual task performance. McCambridge et al. (2016) applied bilateral tDCS with the anode over the right M1 and the cathode over the left M1 and assessed the effects on left-hand and bimanual circle tracing performance. The results indicated a marginal effect on the bimanual task in low performers who received sham stimulation, while no significant effect was found in participants who received active tDCS. Vancleef et al. (2016) examined the effects on task performance of anodal tDCS applied to the left M1 or the left dorsolateral prefrontal cortex (DLPFC) during training on a complex bimanual tracking task (BTT). The BTT requires tracking of a moving dot by rotating two dials with the left (vertical direction) and right (horizontal direction) hands in different frequency-ratios. The authors reported improved motor performance for all participants, independent of tDCS.

Despite the heterogeneity in findings, the data begin to reveal that tDCS may affect relatively easy rather than complex bimanual tasks in particular and that it appears to be more effective in novice volunteers rather than in experts.

\section{tDCS AND BIMANUAL MOTOR PERFORMANCE IN PATIENTS}

Impairment of bimanual skills can occur due to a wide variety of neurological diseases, like stroke or Parkinson's disease, and task performance might be influenced by altered cortical excitability, such as in focal dystonia (FD). In FD, the affected M1 shows pathologically increased activity that is associated with involuntary movements (e.g., tremor) and muscle spasms of the contralateral effector (Cohen and Hallett, 1988; Stinear and Byblow, 2004). Since tDCS likely modulates motor cortical excitability, Furuya et al. (2014b) investigated the effects of five different stimulation configurations (see Table 2 for an overview of different electrode montages) on bimanual task performance in pianists suffering from FD of the right hand and in healthy controls. The study revealed that bilateral tDCS over M1 (affected left M1; cathode over C3, anode over C4) led to increased accuracy of the dystonic hand, both during and after tDCS, while performing metronome-paced symmetrical bimanual finger movements. This effect was not found for bilateral tDCS with the anode over $\mathrm{C} 3$, unilateral tDCS (anode over C4), sham stimulation or-noteworthy for bilateral tDCS - with the cathode over C3 and anode over C4 without concurrent bimanual training. Interestingly, performance gains were positively correlated with FD severity.

Traumatic brain injuries (TBI) can also impair bimanual motor task performance. In a pilot study, Middleton et al. (2014) combined bihemispheric tDCS with physical therapy of the upper extremities in patients suffering from stroke and TBI. Bihemispheric tDCS was applied concurrently with physical therapy for $15 \mathrm{~min}$, with the anode over the ipsilesional M1. The intervention was implemented over 24 sessions, with three sessions per week. The results revealed improved function of the upper extremities in clinical (Fugl-Meyer Assessment 


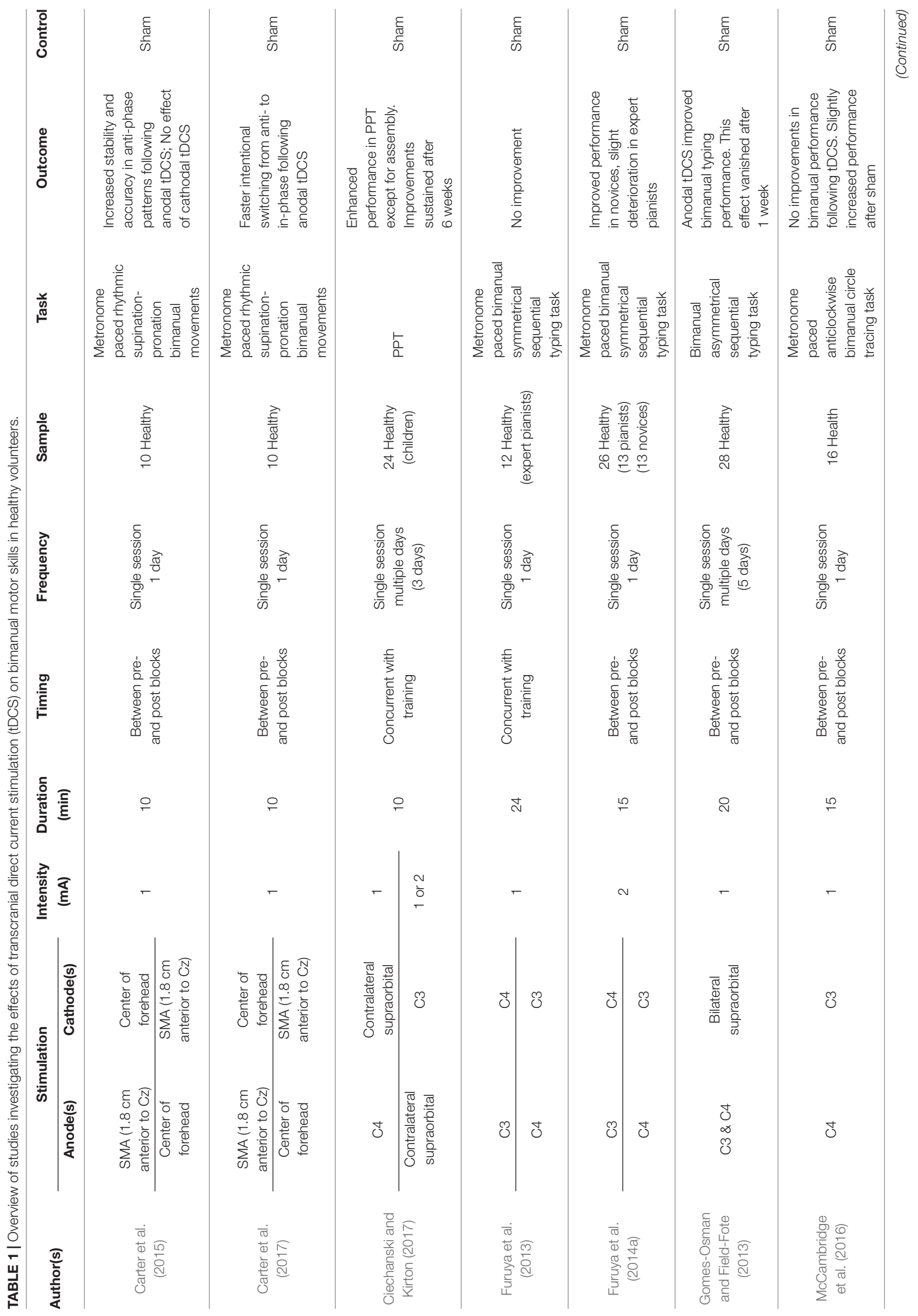




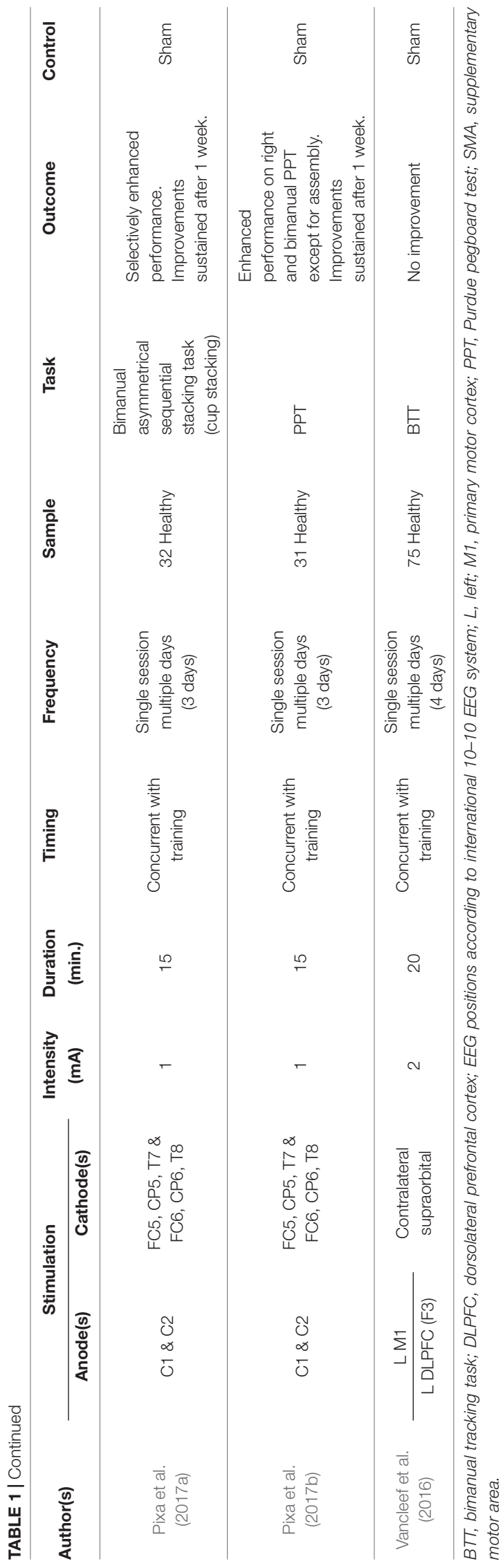

UE, Box and Block Test, PPT, Stroke Impact Scale) and robotic measures (Visually Guided Reaching Task, Object hit task (OHT)). Focusing on bimanual measures, performance in the OHT showed improved bimanual coordination, indicating superior gross motor function. However, bimanual fine motor control in the PPT did not benefit from stimulation. The observed gains persisted up to 6 months. In line with Middleton et al. (2014) the data support the notion that tDCS represents a feasible approach facilitating the effects of physical therapy of the upper extremities. Takeuchi et al. (2012) combined low-frequency, single-pulse, repetitive transcranial magnetic stimulation (rTMS) over the unaffected hemisphere of stroke patients with simultaneous application of anodal tDCS over the affected M1. The combination of rTMS and anodal tDCS was found to reduce motor impairment in these patients (Takeuchi et al., 2012).

\section{DISCUSSION}

In the light of the abundance of everyday tasks requiring bimanual actions and their importance in independent living, the purpose of this review article was to summarize the few existing studies investigating the effects of tDCS on bimanual motor skills. The data suggest that-although some studies have failed to show tDCS-specific effects on bimanual tasks-tDCS has the potential to enhance bimanual motor performance in healthy volunteers as well as in patients suffering from neurological diseases. The overview reveals that-besides the well-known inter- and intra-individual variability of outcome-measures, conflicting study results may be attributed to substantial variations in stimulation protocols, bimanual tasks, and study designs (see Tables 1, 2). Nevertheless, this review article provides a starting point for a systematic evaluation of tDCS effects on bimanual task performance.

Taken together, the data indicate that M1 was the preferred brain area for tDCS application to modulate bimanual performance, in line with the majority of tDCS studies addressing unimanual tasks (for an overview see Buch et al., 2017). However, it must be stressed that the use of stimulation protocols commonly applied for unimanual tasks might represent an oversimplification of the brain processes subserving bimanual task performance since brain areas involved and-more importantly-the temporally precise functional interaction between these areas are assumed to differ between bi- and unimanual tasks (Debaere et al., 2004; Swinnen and Wenderoth, 2004; Wenderoth et al., 2005; Pollok et al., 2007). Several brain regions which are involved in bimanual motor performance are accessible by means of tDCS. The parietal cortex (PC) is suggested to play a pivotal role in bimanual performance through multisensory integration and guidance of movements (Battaglia-Mayer et al., 2006; Buneo and Andersen, 2006), and, learning of bimanual skills (Debaere et al., 2004). Additionally, the right superior temporal gyrus (STG) is proposed to be causally involved in monitoring bimanual spatio-temporal goals (Duque et al., 2010). Furthermore, until now no study had investigated effects of premotor cortex (PMC) or cerebellar tDCS on bimanual motor skills. Moreover, 


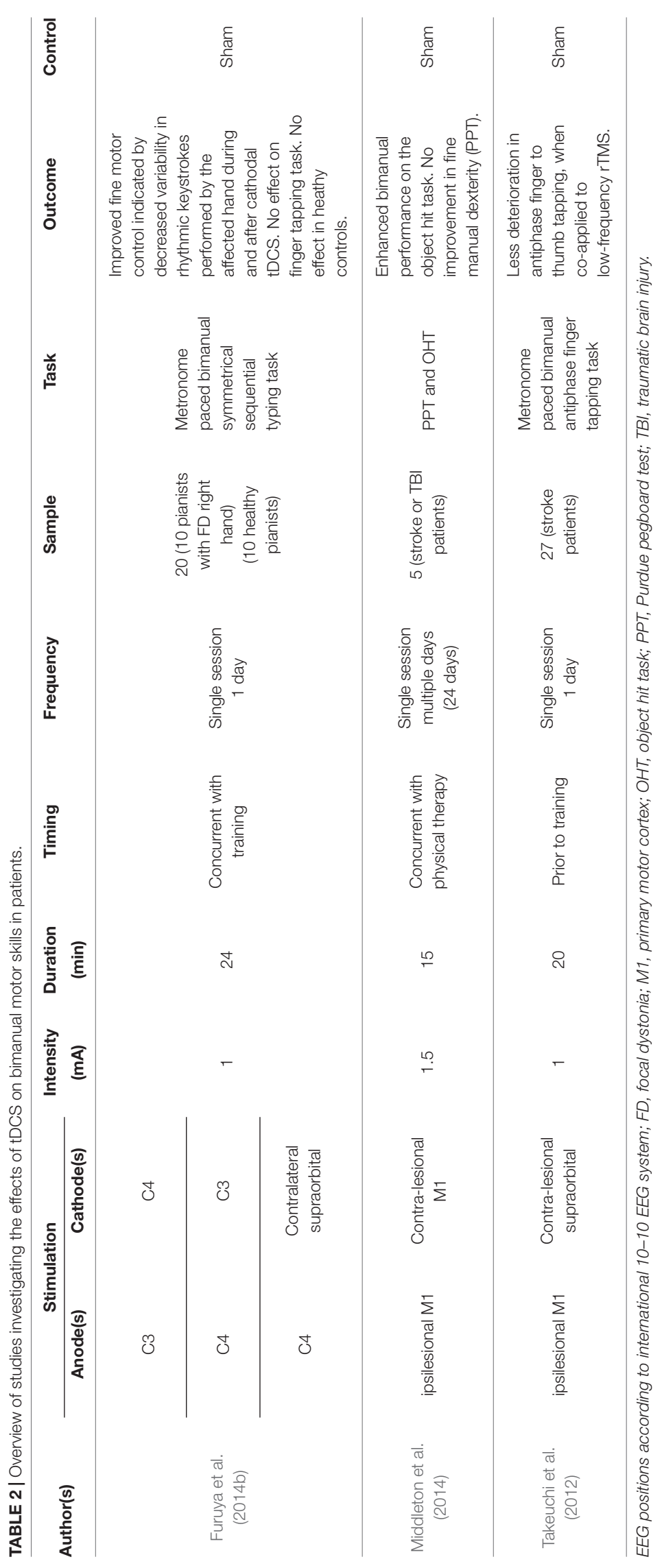


different task demands such as the required type of bimanual action (e.g., symmetric or asymmetric) to achieve a specific task-goal, as well as individual expertise (Furuya et al., 2014a), are likely related to distinct activation patterns within the motor network of bimanual actions (Puttemans et al., 2005; Jantzen et al., 2008; Duque et al., 2010; Whitall et al., 2011). Since complex bimanual tasks are associated with brain activation extending towards the prefrontal, parietal and temporal areas (Gross et al., 2002; Debaere et al., 2004; Hardwick et al., 2013; Swinnen and Gooijers, 2015), the stimulation of a particular brain area might not be effective in modulating complex bimanual skills. This hypothesis fits well with the observation that more complex bimanual tasks remain unaffected by tDCS (Vancleef et al., 2016; Ciechanski and Kirton, 2017; Pixa et al., $2017 a, b)$. Since the neural mechanisms of the wide variety of bimanual actions, as well as the neurophysiological mechanisms underlying tDCS, are not completely understood, future studies need to consider neurophysiological measures using, e.g., TMS, electroencephalography (EEG), magnetoencephalography (MEG) or functional near-infrared spectroscopy (fNIRS). This is particularly important since intra- and inter-individual variability in responses to tDCS is suggested to highly influence study outcomes (Li et al., 2015).

So far, only three studies were identified that have investigated the effects of tDCS on bimanual skills in patients. Although these studies widely differ in terms of the respective stimulation protocol and-even more important-the underlying disease, the findings suggest facilitating effects of tDCS on bimanual task performance. Although sparse, the data imply that tDCS in combination with motor training represents a suitable method for neuro-rehabilitation.

\section{REFERENCES}

Accornero, N., Li Voti, P., La Riccia, M., and Gregori, B. (2007). Visual evoked potentials modulation during direct current cortical polarization. Exp. Brain Res. 178, 261-266. doi: 10.1007/s00221-006-0733-y

Alam, M., Truong, D. Q., Khadka, N., and Bikson, M. (2016). Spatial and polarity precision of concentric high-definition transcranial direct current stimulation (HD-tDCS). Phys. Med. Biol. 61, 4506-4521. doi: 10.1088/00319155/61/12/4506

Alonzo, A., Brassil, J., Taylor, J. L., Martin, D., and Loo, C. K. (2012). Daily transcranial direct current stimulation (tDCS) leads to greater increases in cortical excitability than second daily transcranial direct current stimulation. Brain Stimul. 5, 208-213. doi: 10.1016/j.brs.2011.04.006

Antal, A., Kincses, T. Z., Nitsche, M. A., Bartfai, O., and Paulus, W. (2004). Excitability changes induced in the human primary visual cortex by transcranial direct current stimulation: direct electrophysiological evidence. Invest. Ophthalmol. Vis. Sci. 45, 702-707. doi: 10.1167/iovs. 03-0688

Antal, A., Polania, R., Schmidt-Samoa, C., Dechent, P., and Paulus, W. (2011). Transcranial direct current stimulation over the primary motor cortex during fMRI. Neuroimage 55, 590-596. doi: 10.1016/j.neuroimage.2010. 11.085

Bastani, A., and Jaberzadeh, S. (2012). Does anodal transcranial direct current stimulation enhance excitability of the motor cortex and motor function in healthy individuals and subjects with stroke: a systematic review and meta-analysis. Clin. Neurophysiol. 123, 644-657. doi: 10.1016/j.clinph.2011. 08.029

Batsikadze, G., Moliadze, V., Paulus, W., Kuo, M.-F., and Nitsche, M. A. (2013). Partially non-linear stimulation intensity-dependent effects of direct current
Finally, besides tDCS other techniques like transcranial alternate current stimulation (tACS) or transcranial random noise stimulation (tRNS) are suitable methods for the non-invasive modulation of brain processes subserving motor learning (Prichard et al., 2014; Pollok et al., 2015). However, to the best of our knowledge, no study that had adopted one of these methods in bimanual tasks has been published until yet.

\section{CONCLUSION}

Up until now, knowledge about the effects of tDCS on bimanual performance has remained limited due to a relatively small number of studies with mixed results. However, despite the heterogeneity in stimulation protocols, study designs, and paradigms, the data suggest that tDCS has the potential to enhance bimanual motor performance in healthy volunteers as well as in patients suffering from a variety of neurological diseases. Noteworthy, the data do not allow the identification of specific tDCS parameters as most effective to modulate bimanual motor skills. Therefore, tailoring tDCS protocols to bimanual motor tasks will critically challenge future studies.

\section{AUTHOR CONTRIBUTIONS}

NHP contributed to the conceptualization of the review, performed the literature research, interpretation of the data, wrote the manuscript, approved the final version of the manuscript and acted as corresponding author. BP substantially contributed to the data interpretation, wrote parts of the manuscript and critically revised the manuscript.

stimulation on motor cortex excitability in humans. J. Physiol. 591, 1987-2000. doi: 10.1113/jphysiol.2012.249730

Battaglia-Mayer, A., Archambault, P. S., and Caminiti, R. (2006). The cortical network for eye-hand coordination and its relevance to understanding motor disorders of parietal patients. Neuropsychologia 44, 2607-2620. doi: 10.1016/j. neuropsychologia.2005.11.021

Bindman, L. J., Lippold, O. C. J., and Redfearn, J. W. T. (1962). Long-lasting changes in the level of the electrical activity of the cerebral cortex produced by polarizing currents. Nature 196, 584-585. doi: 10.1038/196584a0

Bindman, L. J., Lippold, O. C. J., and Redfearn, J. W. T. (1964). The action of brief polarizing currents on the cerebral cortex of the rat (1) during current flow and (2) in the production of long-lasting after-effects. J. Physiol. 172, 369-382. doi: 10.1113/jphysiol.1964.sp007425

Buch, E. R., Santarnecchi, E., Antal, A., Born, J., Celnik, P. A., lassen, J., et al. (2017). Effects of tDCS on motor learning and memory formation: a consensus and critical position paper. Clin. Neurophysiol. 128, 589-603. doi: 10.1016/j. clinph.2017.01.004

Buneo, C. A., and Andersen, R. A. (2006). The posterior parietal cortex: sensorimotor interface for the planning and online control of visually guided movements. Neuropsychologia 44, 2594-2606. doi: 10.1016/j.neuropsychologia. 2005.10.011

Carter, M. J., Maslovat, D., and Carlsen, A. N. (2015). Anodal transcranial direct current stimulation applied over the supplementary motor area delays spontaneous antiphase-to-in-phase transitions. J. Neurophysiol. 113, 780-785. doi: 10.1152/jn.00662.2014

Carter, M. J., Maslovat, D., and Carlsen, A. N. (2017). Intentional switches between coordination patterns are faster following anodal-tDCS applied over the supplementary motor area. Brain Stimul. 10, 162-164. doi: 10.1016/j.brs. 2016.11.002 
Ciechanski, P., and Kirton, A. (2017). Transcranial direct-current stimulation can enhance motor learning in children. Cereb. Cortex 27, 2758-2767. doi: 10.1093/cercor/bhw114

Cohen, L. G., and Hallett, M. (1988). Hand cramps: clinical features and electromyographic patterns in a focal dystonia. Neurology 38, 1005-1012. doi: 10.1212/WNL.38.7.1005

Debaere, F., Wenderoth, N., Sunaert, S., van Hecke, P., and Swinnen, S. P. (2004). Changes in brain activation during the acquisition of a new bimanual coodination task. Neuropsychologia 42, 855-867. doi: 10.1016/j. neuropsychologia.2003.12.010

Desrosiers, J., Hébert, R., Bravo, G., and Dutil, E. (1995). The Purdue Pegboard Test: normative data for people aged 60 and over. Disabil. Rehabil. 17, 217-224. doi: 10.3109/09638289509166638

Dieckhöfer, A., Waberski, T. D., Nitsche, M., Paulus, W., Buchner, H., and Gobbelé, R. (2006). Transcranial direct current stimulation applied over the somatosensory cortex-differential effect on low and high frequency SEPs. Clin. Neurophysiol. 117, 2221-2227. doi: 10.1016/j.clinph.2006.06.415

Duque, J., Davare, M., Delaunay, L., Jacob, B., Saur, R., Hummel, F., et al. (2010). Monitoring coordination during bimanual movements: where is the mastermind? J. Cogn. Neurosci. 22, 526-542. doi: 10.1162/jocn.2009.21213

Flöel, A. (2014). tDCS-enhanced motor and cognitive function in neurological diseases. Neuroimage 85, 934-947. doi: 10.1016/j.neuroimage.2013.05.098

Fritsch, B., Reis, J., Martinowich, K., Schambra, H. M., Ji, Y., Cohen, L. G., et al. (2010). Direct current stimulation promotes BDNF-dependent synaptic plasticity: potential implications for motor learning. Neuron 66, 198-204. doi: 10.1016/j.neuron.2010.03.035

Furuya, S., Klaus, M., Nitsche, M. A., Paulus, W., and Altenmuller, E. (2014a). Ceiling effects prevent further improvement of transcranial stimulation in skilled musicians. J. Neurosci. 34, 13834-13839. doi: 10.1523/JNEUROSCI. 1170-14.2014

Furuya, S., Nitsche, M. A., Paulus, W., and Altenmüller, E. (2014b). Surmounting retraining limits in musicians' dystonia by transcranial stimulation. Ann. Neurol. 75, 700-707. doi: 10.1002/ana.24151

Furuya, S., Nitsche, M. A., Paulus, W., and Altenmüller, E. (2013). Early optimization in finger dexterity of skilled pianists: implication of transcranial stimulation. BMC Neurosci. 14:35. doi: 10.1186/1471-2202-14-35

Gomes-Osman, J., and Field-Fote, E. C. (2013). Bihemispheric anodal corticomotor stimulation using transcranial direct current stimulation improves bimanual typing task performance. J. Mot. Behav. 45, 361-367. doi: 10.1080/00222895.2013.808604

Gross, J., Timmermann, L., Kujala, J., Dirks, M., Schmitz, F., Salmelin, R., et al. (2002). The neural basis of intermittent motor control in humans. Proc. Natl. Acad. Sci. U S A 99, 2299-2302. doi: 10.1073/pnas.032682099

Hardwick, R. M., Rottschy, C., Miall, R. C., and Eickhoff, S. B. (2013). A quantitative meta-analysis and review of motor learning in the human brain. Neuroimage 67, 283-297. doi: 10.1016/j.neuroimage.2012.11.020

Ho, K.-A., Taylor, J. L., Chew, T., Gálvez, V., Alonzo, A., Bai, S., et al. (2016). The effect of transcranial direct current stimulation (tDCS) electrode size and current intensity on motor cortical excitability: evidence from single and repeated sessions. Brain Stimul. 9, 1-7. doi: 10.1016/j.brs.2015.08.003

Hunter, M. A., Coffman, B. A., Trumbo, M. C., and Clark, V. P. (2013). Tracking the neuroplastic changes associated with transcranial direct current stimulation: a push for multimodal imaging. Front. Hum. Neurosci. 7:495. doi: 10.3389/fnhum.2013.00495

Jantzen, K. J., Oullier, O., and Scott Kelso, J. A. (2008). Neuroimaging coordination dynamics in the sport sciences. Methods 45, 325-335. doi: 10.1016/j.ymeth. 2008.06.001

Karabanov, A., Ziemann, U., Hamada, M., George, M. S., Quartarone, A., Classen, J., et al. (2015). Consensus paper: probing homeostatic plasticity of human cortex with non-invasive transcranial brain stimulation. Brain Stimul. 8, 442-454. doi: 10.1016/j.brs.2015.01.404

Li, L. M., Uehara, K., and Hanakawa, T. (2015). The contribution of interindividual factors to variability of response in transcranial direct current stimulation studies. Front. Cell. Neurosci. 9:898. doi: 10.3389/fncel.2015.00181

Liebetanz, D., Nitsche, M. A., Tergau, F., and Paulus, W. (2002). Pharmacological approach to the mechanisms of transcranial DC-stimulation-induced after-effects of human motor cortex excitability. Brain 125, 2238-2247. doi: 10.1093/brain/awf238
Maes, C., Gooijers, J., Orban de Xivry, J.-J., Swinnen, S. P., and Boisgontier, M. P. (2017). Two hands, one brain, and aging. Neurosci. Biobehav. Rev. 75, 234-256. doi: 10.1016/j.neubiorev.2017.01.052

McCambridge, A. B., Stinear, J. W., and Byblow, W. D. (2016). Neurophysiological and behavioural effects of dual-hemisphere transcranial direct current stimulation on the proximal upper limb. Exp. Brain Res. 234, 1419-1428. doi: 10.1007/s00221-015-4547-7

Middleton, A., Fritz, S. L., Liuzzo, D. M., Newman-Norlund, R., and Herter, T. M. (2014). Using clinical and robotic assessment tools to examine the feasibility of pairing tDCS with upper extremity physical therapy in patients with stroke and TBI: a consideration-of-concept pilot study. NeuroRehabilitation 35, 741-754. doi: 10.3233/NRE-141178

Monte-Silva, K., Kuo, M.-F., Hessenthaler, S., Fresnoza, S., Liebetanz, D., Paulus, W., et al. (2013). Induction of late LTP-like plasticity in the human motor cortex by repeated non-invasive brain stimulation. Brain Stimul. 6, 424-432. doi: 10.1016/j.brs.2012.04.011

Naros, G., Geyer, M., Koch, S., Mayr, L., Ellinger, T., Grimm, F., et al. (2016). Enhanced motor learning with bilateral transcranial direct current stimulation: impact of polarity or current flow direction? Clin. Neurophysiol. 127, 2119-2126. doi: 10.1016/j.clinph.2015.12.020

Nitsche, M. A., and Paulus, W. (2000). Excitability changes induced in the human motor cortex by weak transcranial direct current stimulation. J. Physiol. 527, 633-639. doi: 10.1111/j.1469-7793.2000.t01-1-00633.x

Nitsche, M. A., and Paulus, W. (2001). Sustained excitability elevations induced by transcranial DC motor cortex stimulation in humans. Neurology 57, 1899-1901. doi: 10.1212/WNL.57.10.1899

Opitz, A., Paulus, W., Will, S., Antunes, A., and Thielscher, A. (2015). Determinants of the electric field during transcranial direct current stimulation. Neuroimage 109, 140-150. doi: 10.1016/j.neuroimage.2015.01.033

Pixa, N. H., Steinberg, F., and Doppelmayr, M. (2017a). Effects of high-definition anodal transcranial direct current stimulation applied simultaneously to both primary motor cortices on bimanual sensorimotor performance. Front. Behav. Neurosci. 11:4506. doi: 10.3389/fnbeh.2017.00130

Pixa, N. H., Steinberg, F., and Doppelmayr, M. (2017b). High-definition transcranial direct current stimulation to both primary motor cortices improves unimanual and bimanual dexterity. Neurosci. Lett. 643, 84-88. doi: 10.1016/j.neulet.2017.02.033

Pollok, B., Boysen, A.-C., and Krause, V. (2015). The effect of transcranial alternating current stimulation (tACS) at $\alpha$ and $\beta$ frequency on motor learning. Behav. Brain Res. 293, 234-240. doi: 10.1016/j.bbr.2015.07.049

Pollok, B., Butz, M., Gross, J., and Schnitzler, A. (2007). Intercerebellar coupling contributes to bimanual coordination. J. Cogn. Neurosci. 19, 704-719. doi: 10.1162/jocn.2007.19.4.704

Prichard, G., Weiller, C., Fritsch, B., and Reis, J. (2014). Effects of different electrical brain stimulation protocols on subcomponents of motor skill learning. Brain Stimul. 7, 532-540. doi: 10.1016/j.brs.2014.04.005

Puttemans, V., Wenderoth, N., and Swinnen, S. P. (2005). Changes in brain activation during the acquisition of a multifrequency bimanual coordination task: from the cognitive stage to advanced levels of automaticity. J. Neurosci. 25, 4270-4278. doi: 10.1523/JNEUROSCI.3866-04.2005

Reis, J., and Fritsch, B. (2011). Modulation of motor performance and motor learning by transcranial direct current stimulation. Curr. Opin. Neurol. 24 , 590-596. doi: 10.1097/WCO.0b013e32834c3db0

Reis, J., Schambra, H. M., Cohen, L. G., Buch, E. R., Fritsch, B., Zarahn, E., et al. (2009). Noninvasive cortical stimulation enhances motor skill acquisition over multiple days through an effect on consolidation. Proc. Natl. Acad. Sci. U S A 106, 1590-1595. doi: 10.1073/pnas.0805413106

Ridding, M. C., and Ziemann, U. (2010). Determinants of the induction of cortical plasticity by non-invasive brain stimulation in healthy subjects. J. Physiol. 588, 2291-2304. doi: 10.1113/jphysiol.2010.190314

Romero Lauro, L. J., Rosanova, M., Mattavelli, G., Convento, S., Pisoni, A., Opitz, A., et al. (2014). TDCS increases cortical excitability: direct evidence from TMS-EEG. Cortex 58, 99-111. doi: 10.1016/j.cortex.2014.05.003

Saucedo Marquez, C. M., Zhang, X., Swinnen, S. P., Meesen, R., and Wenderoth, N. (2013). Task-specific effect of transcranial direct current stimulation on motor learning. Front. Hum. Neurosci. 7:333. doi: 10.3389/fnhum.2013.00333

Stagg, C. J., Best, J. G., Stephenson, M. C., O’Shea, J., Wylezinska, M., Kincses, Z. T., et al. (2009). Polarity-sensitive modulation of cortical neurotransmitters by 
transcranial stimulation. J. Neurosci. 29, 5202-5206. doi: 10.1523/JNEUROSCI. 4432-08.2009

Stagg, C. J., Jayaram, G., Pastor, D., Kincses, Z. T., Matthews, P. M., and JohansenBerg, H. (2011). Polarity and timing-dependent effects of transcranial direct current stimulation in explicit motor learning. Neuropsychologia 49, 800-804. doi: 10.1016/j.neuropsychologia.2011.02.009

Stinear, C. M., and Byblow, W. D. (2004). Impaired modulation of intracortical inhibition in focal hand dystonia. Cereb. Cortex 14, 555-561. doi: 10.1093/cercor/bhh017

Swinnen, S. P. (2002). Intermanual coordination: from behavioural principles to neural-network interactions. Nat. Rev. Neurosci. 3, 348-359. doi: $10.1038 / \mathrm{nrn} 807$

Swinnen, S. P., and Gooijers, J. (2015). "Bimanual coordination," in Brain Mapping: An Encyclopedic Reference, ed. A. W. Toga (Burlington: Elsevier Science), 475-482.

Swinnen, S. P., and Wenderoth, N. (2004). Two hands, one brain: cognitive neuroscience of bimanual skill. Trends Cogn. Sci. 8, 18-25. doi: 10.1016/j.tics. 2003.10.017

Takeuchi, N., Tada, T., Matsuo, Y., and Ikoma, K. (2012). Low-frequency repetitive TMS plus anodal transcranial DCS prevents transient decline in bimanual movement induced by contralesional inhibitory rTMS after stroke. Neurorehabil. Neural Repair 26, 988-998. doi: 10.1177/1545968311433295

Tiffin, J., and Asher, E. J. (1948). The Purdue pegboard; norms and studies of reliability and validity. J. Appl. Psychol. 32, 234-247. doi: 10.1037/h0061266

Vancleef, K., Meesen, R., Swinnen, S. P., and Fujiyama, H. (2016). tDCS over left M1 or DLPFC does not improve learning of a bimanual coordination task. Sci. Rep. 6:35739. doi: 10.1038/srep35739

Villamar, M. F., Volz, M. S., Bikson, M., Datta, A., DaSilva, A. F., and Fregni, F. (2013). Technique and considerations in the use of $4 \mathrm{x} 1$ ring high-definition transcranial direct current stimulation (HD-tDCS). J. Vis. Exp. 77:e50309. doi: $10.3791 / 50309$

Wenderoth, N., Debaere, F., Sunaert, S., and Swinnen, S. P. (2005). Spatial interference during bimanual coordination: differential brain networks associated with control of movement amplitude and direction. Hum. Brain Mapp. 26, 286-300. doi: 10.1002/hbm.20151

Whitall, J., Waller, S. M., Sorkin, J. D., Forrester, L. W., Macko, R. F. Hanley, D. F., et al. (2011). Bilateral and unilateral arm training improve motor function through differing neuroplastic mechanisms: a single-blinded randomized controlled trial. Neurorehabil. Neural Repair 25, 118-129. doi: $10.1177 / 1545968310380685$

Woods, A. J., Antal, A., Bikson, M., Boggio, P. S., Brunoni, A. R., Celnik, P., et al. (2016). A technical guide to tDCS, and related non-invasive brain stimulation tools. Clin. Neurophysiol. 127, 1031-1048. doi: 10.1016/j.clinph.2015.11.012

Zaehle, T., Beretta, M., Jäncke, L., Herrmann, C. S., and Sandmann, P. (2011). Excitability changes induced in the human auditory cortex by transcranial direct current stimulation: direct electrophysiological evidence. Exp. Brain Res. 215, 135-140. doi: 10.1007/s00221-011-2879-5

Conflict of Interest Statement: The authors declare that the research was conducted in the absence of any commercial or financial relationships that could be construed as a potential conflict of interest.

Copyright (c) 2018 Pixa and Pollok. This is an open-access article distributed under the terms of the Creative Commons Attribution License (CC BY). The use, distribution or reproduction in other forums is permitted, provided the original author(s) and the copyright owner are credited and that the original publication in this journal is cited, in accordance with accepted academic practice. No use, distribution or reproduction is permitted which does not comply with these terms. 